\title{
Genomic landscape of Juvenile Myelomonocytic Leukemia (JMML) - A real world context
}

\author{
SHRINIDHI NATHANY ${ }^{1}$, Gaurav Chatterjee ${ }^{1}$, Shruti Ghai ${ }^{1}$, Nirmalya Roy Moulik ${ }^{1}$, \\ Dhanlaxmi Shetty ${ }^{1}$, Papagudi Subramanian ${ }^{1}$, Prashant Tembhare ${ }^{1}$, Sumeet Gujral ${ }^{2}$, \\ Chetan Dhamne ${ }^{2}$, Sripad Banavali ${ }^{1}$, Gaurav Narula ${ }^{1}$, and Nikhil Patkar ${ }^{1}$ \\ ${ }^{1}$ Tata Memorial Centre \\ ${ }^{2}$ Tata Memorial Hospital
}

June 4, 2020

\begin{abstract}
Background: JMML is a pediatric haematopoietic stem cell malignancy characterised by uncontrolled proliferation of myelomonocytic and progenitor compartments and a poor outcome. We comprehensively evaluated the genomic profile of JMML that presented to our hospital for diagnosis and treatment. Procedure: We developed a 51-gene (151.5kB) low-cost targeted myeloid panel based on single-molecule molecular inversion probes. A total of 50 children with clinical and pathological features of JMML were sequenced at high coverage on an Illumina MiSeq. The presenting clinical, laboratory and follow-up data were procured from the electronic medical record system of the institution. Results: The median age of our cohort was 2 years, with a male preponderance. Among the 50 patients, $43(86 \%)$ harboured mutations in one of the RAS/MAPK-pathway genes, most frequently in PTPN11 (14, 28\%), and NRAS (14, 28\%), followed by NF1(11. 22\%). Interestingly, 20\% (10) of children had more than one mutation, with 5 cases harbouring two RAS-pathway mutations. Monosomy 7 was detected in 32\% (16) patients, and five of these did not harbour any RAS-pathway mutations. The follow-up data revealed that $37(74 \%)$ of these children had succumbed to the disease. Children with monosomy 7 showed shorter overall survival, compared to their wildtype counterparts $(\mathrm{p}=0.02)$. Conclusion: Our study highlights that comprehensive genomic profiling identifies at least one mutation in almost $90 \%$ of JMML patients. Performing genomic analysis early in evaluation of JMML might help in triaging patients for allogenic stem cell transplant in resource-constrained settings.
\end{abstract}

\section{INTRODUCTION}

Juvenile myelomonocytic leukemia (JMML) is a rare childhood neoplasm ( $<5 \%$ cases), which has been categorised under myelodysplastic/myeloproliferative neoplasms (MDS/MPN) in the recent classification by the World Health Organisation(1). It is characterised by a wide spectrum of clinical manifestations and is rapidly fatal if not appropriately treated (2). The only current curative therapy for non-syndromic de novo JMML patients is hematopoietic stem cell transplantation (HSCT) and the clinical and pathologic factors are not sufficient to predict the prognostic heterogeneity observed among JMML patients(3).

Molecular diagnosis assumes paramount importance in diagnosis of JMML as it lacks definite clinical features and morphological dysplasia, seen in other adult MDS/MPN. Moreover, flowcytometric and karyotyping abnormalities are present in only a subset of cases (4). It has been reported in many studies that mutations involving one of the genes in the RAS pathway, (NRAS, KRAS, c-CBL, PTPN11 andNF1) are present in $85 \%$ of these cases $(5,6)$. Yet, around $15 \%$ cases of JMML do not depict any molecular aberration in these five cardinal genes, which underscores the need of high-throughput techniques with larger panels to uncover the full mutational spectrum of JMML. Molecular evaluation could also aid in risk stratification of these patients with respect to the need of hematopoietic stem cell transplant $(\mathrm{HSCT})(7,8)$. This type of 
molecular triaging is especially relevant with respect to the Indian subcontinent where economic constraints prevail in a large section of the population.

In this study, we comprehensively evaluated the genomic profile of JMML that were referred to our hospital for diagnosis and treatment

\section{METHODS}

1. Clinical Information and treatment protocol: Fifty (50) children with JMML confirmed on the basis ofWHO diagnostic criteria were included in this study. Peripheral blood/bone marrow aspirate samples of these patients procured as a part of the routine diagnostic workup were retrieved from the sample archive of our laboratory. The clinical information, other laboratory findings and follow up data were obtained from the electronic medical record system of the institution. No extra samples or interventional procedures were undertaken or performed as a part of this study. Children with diagnosed JMML were started on work-up for bone marrow transplant immediately on diagnosis. Oral combination chemotherapy including 6 -mercaptopurine $(6 \mathrm{MP})(50 \mathrm{mg} / \mathrm{m} 2)$ or thioguanine (6TG) $(40 \mathrm{mg} / \mathrm{m} 2)$, etoposide $(50 \mathrm{mg} / \mathrm{m} 2)$ and cis-retinoic acid $(100 \mathrm{mg} / \mathrm{m} 2)$ were started in the interim for symptomatic patients with the aim to halt progression /transformation till the time transplant could be done. However, patients for whom transplant did notmaterialize due to lack ofresources/donor, the same combination chemotherapy was continued indefinitely, aiming for long term symptom control.

2. Samples The samples used for this assay were peripheral blood/bone marrow aspirate samples, from which DNA was extracted using PureGeneGentra manual protocol (Qiagen) for DNA and automated RNA extraction using the Qiacube (Qiagen). The quality and quantity of the extracted nucleic acid was checked using Nanodrop spectrophotometry (Thermo Fischer Scientific Inc) and Qubit Fluorometry (Thermo Fischer Scientific Inc).

3. Next generation sequencing assay

1. Detection of mutations using single molecule molecular inversion probes (smMIPS)We performed next generation sequencing assay using a targeted gene panel comprising of 50 genes, covered in 1066 probes, based on thesmMIPS principle $(9,10)$. The genomic footprint of the panel was $151.5 \mathrm{~kb}$. The 50 genes included in this panel, are known to be commonly implicated in myeloid malignancies, and based on the available data on COSMIC (Catalogue of Somatic Mutations in Cancer) and published literature, the hotspot regions in the coding regions of these genes were identified(Supporting information Table S1). These 1066 probes capture the coding regions in these genes with a 5bp flank covering the introns on either side. These 1066 probes were initially pooled in equimolar concentrations in 4 different pools and these pools were subsequently balanced to ensure uniform coverage across the entire panel, indicating optimum capture efficiency. For the samples, 600ng DNA was used as starting material, and after a 24-hour capture reaction, exonuclease treatment was performed, followed by amplification by polymerase chain reaction (PCR), incorporating dual sample specific indices. After ensuring adequate amplification and quality checks at each step, a magnetic bead clean up and size selection were performed which generated sequencing ready fragments. These samples were then sequenced on an Illumina Miseq using the v2-300 cycle chemistry.

2. Informatics and analysis of the sequenced dataThe Fastq files which were generated were then demultiplexed using the MiSeq instrument demultiplexing software. Paired end assembly was performed using PEAR (v0.9.8). The assembled reads were mapped to the human genome (build hg19) using burrows wheeler alignment software (v0.7.17) and further preprocessing was done using Picard (v2.1.1) and samtools (v1.3.1). Alignment files were further processed using different GATK (v3.8) walkers (RealignerTargetCreator, IndelRealigner, BaseRecalibrator). A. mpileup file was generated using samtools (v1.3.1). Variant calling was performed using Mutect2, Platypus (v.0.8.1) and Varscan2 (v.2.3.9) and Vardict. Variant files were annotated using annovar with population frequency databases (1000Genomes, Exome Aggregation Consortium) as well as the COSMIC database (Cosmic v83). This data was processed and collated with internal scripts. Variants were filtered by focusing on exonic regions (including splicing variants if any) followed by nonsynonymous and population frequency $(<0.01)$ 
filtering. This was followed by in-silico prediction using multiple tools (SIFT, PolyPhen2, CADD, PROVEAN, MutationTaster, MutationAssessor, M-CAP, FATHMM, LRT, DANN).

\section{4) Statistical methods used for Analysis of Data}

The data was analysed using MedCalc QC software (version 14.8.1, MedCalc, Ostend, Belgium). Descriptive statistics using measures of central tendency were employed to determine median and mean values. Clinical and biologic features were compared between groups using $\chi 2$ test (for categorical variables) or $\mathrm{t}$ test (for continuous variables), and Fisher's exact test when data were sparse. The impact of an individual parameter on overall survival (OS) was detected using Kaplan-Meier Technique.

\section{RESULTS}

Demographic, clinical and laboratory features

We recruited fifty cases of already diagnosed JMML, who had attended our facility. The median age of our cohort was 2years (range: 1.2-84 months), with a male preponderance; male to female ratio being 2.6:1 (36 males, 14 females). Clinically, dysmorphic facial features were observed in 2 cases, who did not harbour a syndromic PTPN11 mutation, and 4 cases had café au lait spots on their limbs and back, and all four had NF1 mutation, indicating a syndromic association (Table 1). The median hemoglobin concentration of the entire cohort was $8.8 \mathrm{~g} / \mathrm{dL}(3.5-13.6 \mathrm{~g} / \mathrm{dl})$. The median platelet count was $44.5 \times 10^{9} / \mathrm{L}\left(6-227 \times 10^{9} / \mathrm{L}\right)$. The median peripheral blood absolute monocyte count was $2.7 \times 10^{9} / \mathrm{L}\left(1-13.2 \times 10^{9} / \mathrm{L}\right)$ and the median peripheral blood blast count was $5 \%(0-16 \%)$. When baseline clinical and laboratory parameters were compared, children with one molecular alteration were found to be younger $(<2$ years, $\mathrm{p}=0.001)$ and had less peripheral blood blast percentage $(<5 \%, \mathrm{p}=0.013)$ as compared to children with $>1$ molecular alteration (Table 2$)$.

Bone marrow morphology and Immunophenotype

Results of bone marrow studies were available in 42 cases as the others $(n=8)$ were diagnosed elsewhere(Table 1). Flowcytometric immunophenotypic evaluation was performed in 40 cases. Most of the cases $(\mathrm{n}=37)$ showed abnormal myeloid blasts and dyspoiesis in the monocyte and granulocyte lineages. A representative flow cytometric evaluation study of a childdepicting these abnormalities is shown in Figure1.

Next Generation Sequencing

We performed targeted panel based next generation sequencing on fifty samples from patients who were diagnosed as JMML on the basis of clinical and pathological findings. At least one molecular alteration was present in $44(88 \%)$ of these children. Mutations in at least one of the RAS pathway genes were encountered in $43(86 \%)$ of these patients. The most frequently mutated genes in our cohort were $N R A S \quad(16,32 \%)$, and PTPN11 (14, 28\%), followed by NF1 mutation in 22\% (11) cases (Figure 2A).KRAS and CBL were mutated in $5(10 \%)$ and $1(2 \%)$ children. Most frequent non-RAS pathway gene to be mutated was ASXL1 $(3.6 \%)$.

Interestingly, $20 \%$ (10) of children had more than one mutation, with 5 cases harbouring two RAS pathway mutations. Of these 5 patients, one patient harboured two coexistent mutations in the NF1 gene, three patients had coexistent PTPN11 and NF1 mutations and one patient had a coexistent NF1 and NRAS mutations. Additionally, two patients also had an ASXL1 mutation, coexistent withNF1 and NRAS mutations respectively. Other non-RAS pathway mutations involved ABL1, ATRX, SETBP1, SH2B3 andZRSR2 genes. (Figure 2B) The median variant allele frequency of RAS pathway mutations detected was $40.75 \%$.

Monosomy 7 was detected in $32 \%$ (16) children, and six of these did not harbour any RAS pathway mutations. The five patients who did not harbour any mutations, showed monosomy 7 on cytogenetics analysis by FISH and conventional karyotyping, hence meeting the WHO criteria for diagnosis of JMML. Additionally, an overview of all the mutations encountered, with number of mutations per patient, the type of mutation (missense, indel), the variant allele frequency and co-occurrence of mutations has been depicted in Figure $2 \mathrm{~A}$ and $2 \mathrm{~B}$. 


\section{NRAS mutations}

NRAS mutation was the most commonly encountered mutation, detected in $32 \%$ cases. These were clustered mainly in the G12, G13 and Q61 hotspots of exons 2 and 4 of the NRAS gene.Two of these cases, harboured additional mutations: One child with an additional NF1 mutation, and the other with NF1 and ASXL1 mutations

\section{PTPN11 mutations}

PTPN11 mutation was detected in $28 \%$ cases, with most of them located in the E76 (4), A72 (3), D69 (3), D61(2) codons. Two patients showed a duplication in the 503 codon. However, none of the patients clinically revealed any Noonan-like features.

Follow up and Outcome

The follow up data revealed that $34(68 \%)$ of these patients had succumbed to the disease. This dismal outcome could be attributed to only five children receiving allogenic stem cell transplant, owing to economic constraints; and two of these five patients had succumbed to the disease. The median time period from diagnosis to death was 7.2 months in the cohort. The follow-up and outcome findings are depicted in Figure 3 .

On survival analysis, none of the individual molecular mutation was associated with significant change in OS. However, children with monosomy 7 showed a trend to shorter overall survival, compared to their wild type counterparts $(p=0.02$, median OS 6.33 months with a range of 4.2-12.5 months vs 8.6 months with a range of 11.0-19.9 months) (Figure 4). Additionally, children who harboured more than one molecular alteration tend to have inferior overall survival with median OS of 7.4 months (5.241-9.179 months) vs 8.1 months (9.265-28.229 months), however this association did not reach statistical significance. Among the two children who succumbed to the disease despite receiving allogenic stem cell transplant, one child had coexistent NRAS mutation with monosomy 7, and the other child harboured multiple mutations inPTPN11 and NRAS. The three children who are doing good without relapse/progression afterallogenic stem cell transplant harboured mutation in NF1, NF1 and CBL respectively without monosomy 7.

Among the thirteen patients who are still on regular follow-up, two patients have relapsed with disease progression to acute myeloid leukemia and $\mathrm{T} /$ myeloid mixed phenotypic acute leukemia (MPAL) respectively. The patient who has progressed to acute myeloid leukemia, harboured NRAS mutation at primary diagnosis of JMML with $<20 \%$ blasts on peripheral blood and bone marrow. This patient relapsed as AML after a period of 23 months, during which he received etoposide based chemotherapy. Transplant could not be performed owing to economic constraints. At relapse, genomics could not be performed and this child succumbed to the disease after 3 months. The other patient who relapsed as mixed phenotype acute leukemia (T/myeloid) had mutations in the PTPN11 and ZRSR2 genes at diagnosis. The mutation in ZRSR2 might have been a germline polymorphism as the mother of the patient also tested positive for the same variant encountered in the patient. He relapsed after a period of 11 months, and targeted next generation sequencing performed at relapse revealed a subclonal TP53 mutation, and the same ZRSR2 mutation detected at baseline. The PTPN11 mutation was not detected at relapse.

\section{DISCUSSION}

JMML remains a rare childhood malignancy with dismal outcome, with only curative treatment being allogenic stem cell transplant. Although mutation in one of the cardinal five RAS pathway genes in JMML has been elucidated extensively $(5,6,11)$, the broader genetic spectrum of this disease has only recently been started to unravel $(8,12)$. Our cohort represents one of the largest single centre experiences and is the first of such studies fromthe Indian subcontinent.

The median age of our population was 2 years with $88 \%$ children harbouring atleast one gene mutation and the rest had monosomy 7 . Our finding of at least one RAS pathway mutation in $86 \%$ of children with JMML is similar to that reported byStieglitz et al(8). In our cohort,PTPN11 mutations were detected in 
$28 \%$ cases, of which none had any clinical features suggestive of Noonan's syndrome. It has been reported that non-syndromic somatic mutations in PTPN11 gene can be found in $35 \%$ cases with the most common mutations occurring in the E76 codon(13). In our cohort, however, germline testing was not performed in all 14 cases, to confirm the somatic nature of the mutation. In six of these patients, the samples from both the parents of the proband were tested, which yielded negative results. Additionally, the patient who relapsed as MPAL(T/myeloid) had a PTPN11 mutation at baseline, which was not detected at relapse, confirming that it was not present in germline.

There were $10 \%$ children who harboured more than 1 mutation, with two concurrent mutations in NF1 and NF1 with PTPN11 mutations being the most common combinations. Mutations in more than one driver gene have been reported in $11 \%$ cases in literature, and are associated with an overall adverse outcome(7, 8). Additionally, we found that these patients tend to be older when compared to those with 1 molecular alteration, and also show more than $5 \%$ blasts in peripheral blood. The association of $>1$ molecular alteration with higher peripheral blood blast count has not been widely elucidated in literature. The plausible explanation for this could be the higher leukemogenic potential imparted by RAS pathway genes, which has been previously documentedin AML cases(14).

We also identified mutations in genes involved in gene splicing and polycomb repressive complex 2 (PRC2). One patient had a missense mutation in the SH2B3 gene, which has been reported in $7 \%$ cases previously, with the potential of leukemic transformation $(8,15)$. Our case however, had a missense exon 8 mutation which was predicted to be of uncertain significance using in-silico prediction tools, and on follow up is currently disease free for last 6 months. Two patients also harboured an additional ASXL1 mutation which has also been documented in JMML patients, and known to portend an adverse outcome in these cases(16). Spliceosome complex gene mutation involving theZRSR2 gene was found in only one patient, which was most likely a germline polymorphism, as itwas also detected in the patient's mother. This patient later on relapsed as MPAL (T/myeloid) and retained theZRSR2 mutation.

A multitude of studies have started to find association with molecular aberration and outcome in children with JMML. One of the most consistent observations has been adverse outcome in children with PTPN11 mutations and these children generally require transplant $(17,18)$. More recently, children with higher number of molecular alterationshave been shown to have poorer outcome (8). Furthermore, recent studies have shown that DNA methylation studies could identify children with distinct outcome (19). In our cohort, only monosomy 7 was associated with poorer OS ( $p=0.02$, median OS 6.33 months with a range of 4.279-12.5 months vs 8.6 months with a range of 11.026-19.996 months). Children with $>1$ molecular alteration did show a trend of inferior OS, but this association did not reach statistical significance. The lack of association of individual molecular alteration and significant change in outcome could be explained by the overall poorer outcome in our cohort, which is attributable to only a few children $(5 / 50)$ receiving allogenic stem cell transplant due to economic constraints. Furthermore, we found out that two children, who succumbed to the disease even after receiving transplant, had high-risk molecular features like PTPN11 mutation, monosomy 7 and harboured more than one molecular alteration. On the contrary, three children who did not progress/relapse after transplant, had single genetic lesion in our panel. This data seems to indicate that molecular triaging performed at baseline may help in selecting the children most likely to benefit in a resource-constrained setting.

This study is the largest single centre experience from Indian subcontinent and highlights broad genomic landscape of JMML using a fairly large targeted next-generation sequencing panel. Our studyhighlights that comprehensive genomic profiling identifies at least one mutation in almost $90 \%$ of JMML patients.Performing genomic analysis early in evaluation of JMML might help in triaging patients for allogenic stem cell transplant in resource-constrainedsettings.

\section{CONFLICT OF INTEREST STATEMENT: None}

ACKNOWLEDGEMENT: We acknowledge the support from departments of Pediatric and Medical Oncology of Tata Memorial Hospital, Mumbai.This work was supported by the Wellcome Trust/DBT India 
Alliance Fellowship [grant number IA/CPHI/14/1/501485] awarded to Dr Nikhil Patkar.

Data availability statement: The data that support the findings of this study are available from the corresponding author upon reasonable request.

\section{REFERENCES:}

1. Arber DA, Orazi A, Hasserjian R, Thiele J, Borowitz MJ, Le Beau MM, et al. The 2016 revision to the World Health Organization classification of myeloid neoplasms and acute leukemia. Blood. 2016;127(20):2391405.

2. Dvorak CC, Loh ML. Juvenile myelomonocytic leukemia: molecular pathogenesis informs current approaches to therapy and hematopoietic cell transplantation. Frontiers in pediatrics. 2014;2:25.

3. Locatelli F, Niemeyer CM. How I treat juvenile myelomonocytic leukemia (JMML). Blood. 2015.

4. Niemeyer CM, Arico M, Basso G, Biondi A, Cantu Rajnoldi A, Creutzig U, et al. Chronic myelomonocytic leukemia in childhood: a retrospective analysis of 110 cases. European Working Group on Myelodysplastic Syndromes in Childhood (EWOG-MDS). Blood. 1997;89(10):3534-43.

5. Niemeyer CM. RAS diseases in children. Haematologica. 2014;99(11):1653-62.

6. de Vries ACH, Zwaan CM, van den Heuvel-Eibrink MM. Molecular basis of juvenile myelomonocytic leukemia. Haematologica. 2010;95(2):179-82.

7. Koegel AS, E. A Risk Stratified Treatment Algorithm for Patients with Juvenile Myelomonocytic Leukemia. American Society of Hematology. 2017;14(2).

8. Stieglitz E, Taylor-Weiner AN, Chang TY, Gelston LC, Wang Y-D, Mazor T, et al. The genomic landscape of juvenile myelomonocytic leukemia. Nature Genetics. 2015;47:1326.

9. Patkar N, Shaikh AF, Kakirde C, Nathany S, Ramesh H, Bhanshe P, et al. A novel machine-learningderived genetic score correlates with measurable residual disease and is highly predictive of outcome in acute myeloid leukemia with mutated NPM1. Blood Cancer Journal. 2019;9(10):79.

10. Hiatt JB, Pritchard CC, Salipante SJ, O'Roak BJ, Shendure J. Single molecule molecular inversion probes for targeted, high-accuracy detection of low-frequency variation. 2013;23(5):843-54.

11. Niemeyer CM, Kang MW, Shin DH, Furlan I, Erlacher M, Bunin NJ, et al. Germline CBL mutations cause developmental abnormalities and predispose to juvenile myelomonocytic leukemia. Nat Genet. 2010;42(9):794-800.

12. Chao AK, Meyer JA, Lee AG, Hecht A, Tarver T, Van Ziffle J, et al. Fusion driven JMML: a novel CCDC88C-FLT3 fusion responsive to sorafenib identified by RNA sequencing. Leukemia. 2020;34(2):662-6.

13. Kratz CP, Niemeyer CM, Castleberry RP, Cetin M, Bergstrasser E, Emanuel PD, et al. The mutational spectrum of PTPN11 in juvenile myelomonocytic leukemia and Noonan syndrome/myeloproliferative disease. Blood. 2005;106(6):2183-5.

14. Ward AF, Braun BS, Shannon KM. Targeting oncogenic Ras signaling in hematologic malignancies. Blood. 2012;120(17):3397-406.

15. Caye A, Strullu M, Guidez F, Cassinat B, Gazal S, Fenneteau O, et al. Juvenile myelomonocytic leukemia displays mutations in components of the RAS pathway and the PRC2 network. Nat Genet. 2015;47(11):133440.

16. Perez B, Kosmider O, Cassinat B, Renneville A, Lachenaud J, Kaltenbach S, et al. Genetic typing of CBL, ASXL1, RUNX1, TET2 and JAK2 in juvenile myelomonocytic leukaemia reveals a genetic profile distinct from chronic myelomonocytic leukaemia. British journal of haematology. 2010;151(5):460-8. 
17. Loh ML, Reynolds MG, Vattikuti S, Gerbing RB, Alonzo TA, Carlson E, et al. PTPN11 mutations in pediatric patients with acute myeloid leukemia: results from the Children's Cancer Group. Leukemia. 2004;18(11):1831-4.

18. Chan RJ, Cooper T, Kratz CP, Weiss B, Loh ML. Juvenile myelomonocytic leukemia: a report from the 2nd International JMML Symposium. Leukemia research. 2009;33(3):355-62.

19. Stieglitz E, Mazor T, Olshen AB, Geng H, Gelston LC, Akutagawa J, et al. Genome-wide DNA methylation is predictive of outcome in juvenile myelomonocytic leukemia. Nature communications. 2017;8(1):2127.

\section{FIGURE LEGENDS}

FIGURE 1 Flow cytometry plots of a representative case. 1A: CD45 vs SSC gating plot shows the CD45 dim blast population (colored in red). 1B: Progenitor gate: Blasts are positive for CD34 and CD117. 1C: HLA-DR positivity in the blasts. 1D: CD33 in a subset of the blast population. 1E: Aberrant CD7 expression in the myeloid blast population. 1F: Aberrant CD7 expression in monocytes. 1G: Asynchronous maturation pattern in monocytes on CD14 vs HLA DR plot. 1H: Asynchronous maturation in granulocytes in CD13 vs CD16 plot

FIGUE 2 Molecular spectrum of JMML in the cohort. 2A:Oncoplot depicting various mutations detected on next generation sequencing; 2B: Circos plot depicting co-occurring mutations

FIGURE 3 Outcome and follow up of the children with JMML

FIGURE 4 Survival analysis of children with JMML with respect to presence of monosomy 7

\section{Hosted file}

Table 1.docx available at https://authorea.com/users/329791/articles/456713-genomiclandscape-of-juvenile-myelomonocytic-leukemia-jmml-a-real-world-context

\section{Hosted file}

Table 2.docx available at https://authorea.com/users/329791/articles/456713-genomiclandscape-of-juvenile-myelomonocytic-leukemia-jmml-a-real-world-context
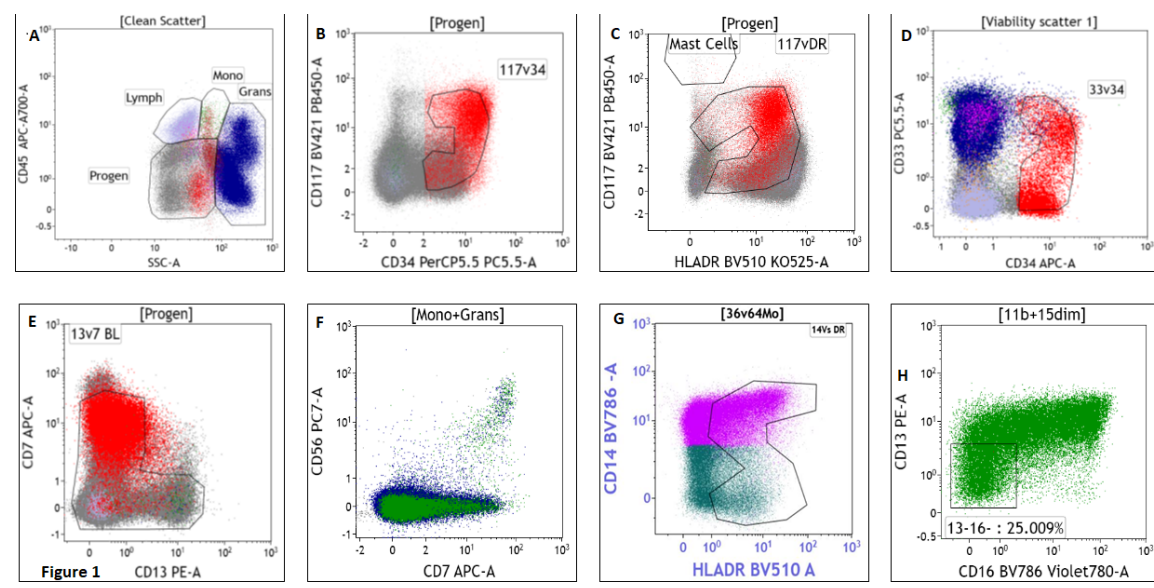
A

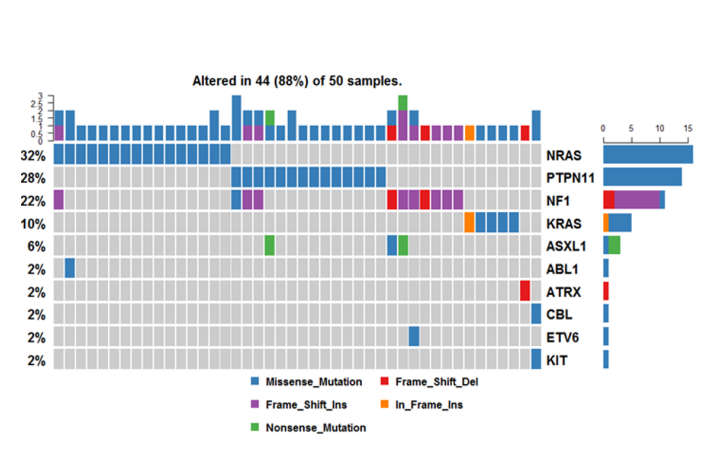

B

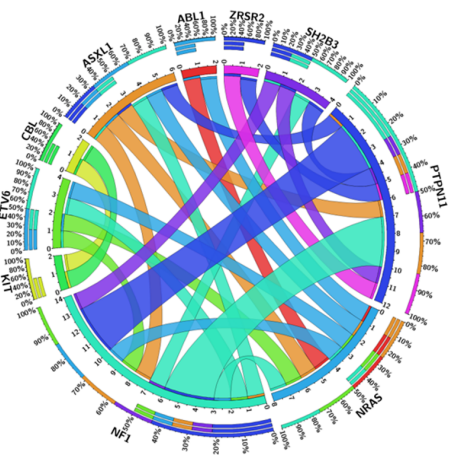

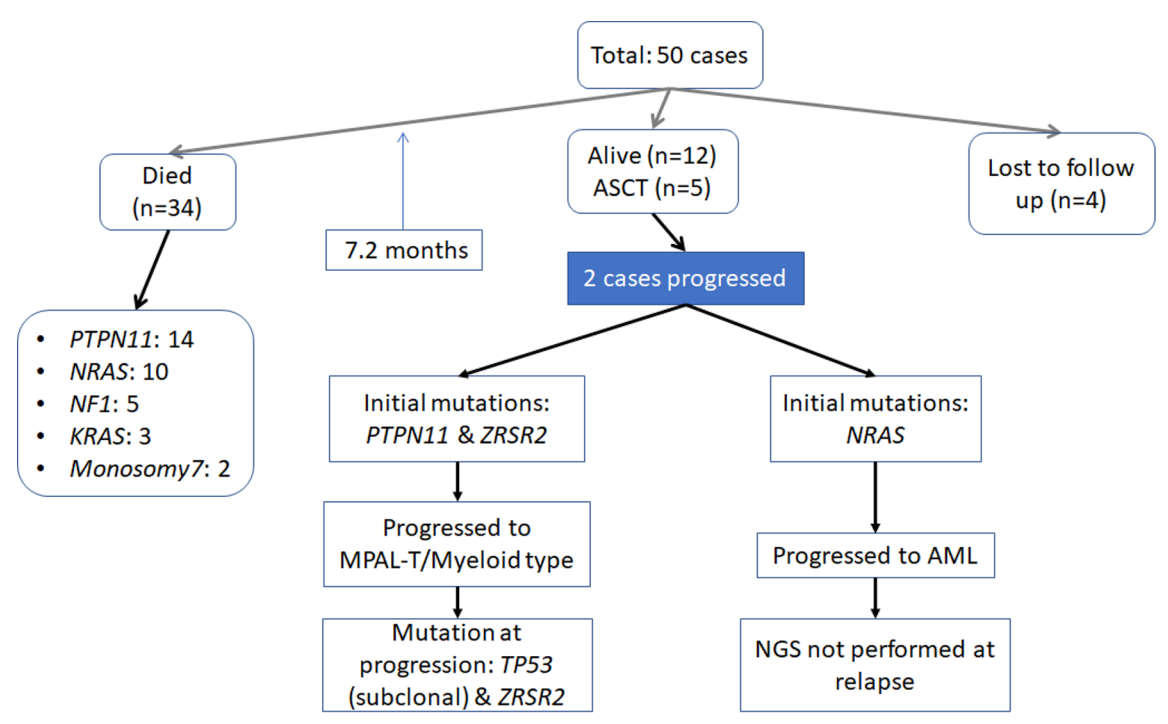




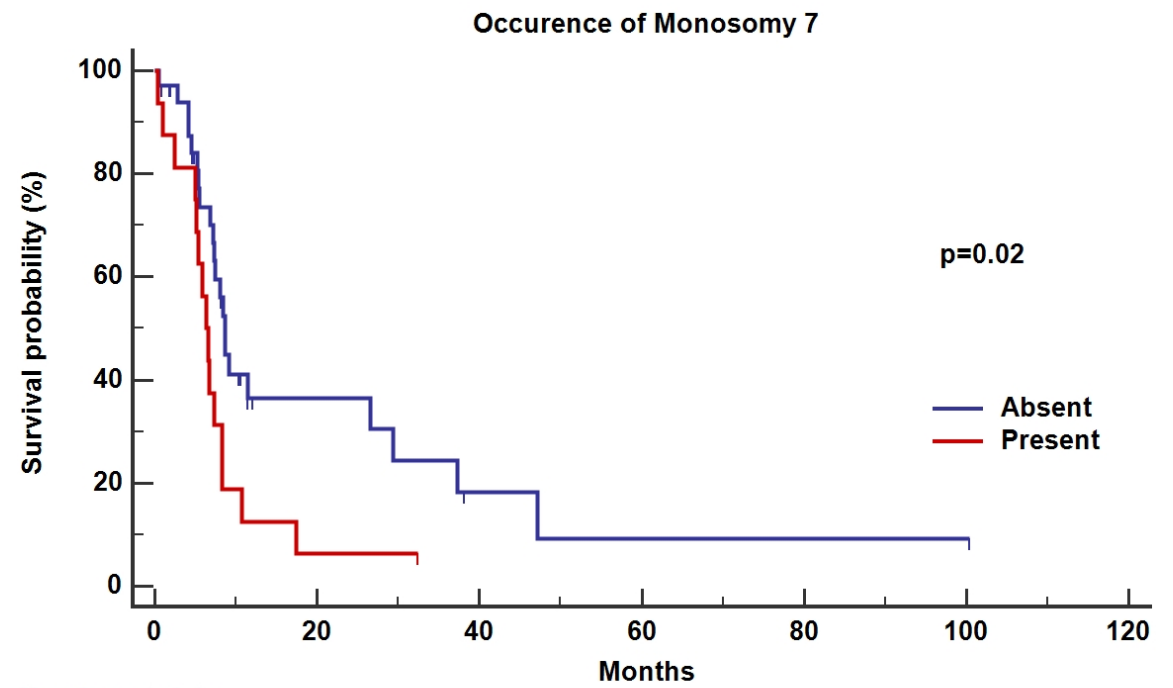

Number at risk Group: Absent

Group: Present

16

6

1

2
0

1
0

0

1
0

1

0
0

0 